Cahiers

Claude Simon

\section{Cahiers Claude Simon}

13 | 2018

Le Jardin des Plantes

\title{
L'enregistrement du Jardin Des Plantes
}

The Recording in Le Jardin Des Plantes

\section{Didier Alexandre}

\section{(2) OpenEdition}

Journals

Édition électronique

URL : http://journals.openedition.org/ccs/1521

DOI : $10.4000 /$ ccs.1521

ISSN : 2558-782X

Éditeur :

Presses universitaires de Rennes, Association des lecteurs de Claude Simon

Édition imprimée

Date de publication : 30 août 2018

ISBN : 978-2-7535-7489-2

ISSN : 1774-9425

\section{Référence électronique}

Didier Alexandre, "L'enregistrement du Jardin Des Plantes », Cahiers Claude Simon [En ligne], 13 | 2018, mis en ligne le 30 août 2019, consulté le 20 février 2020. URL : http://journals.openedition.org/ccs/ 1521 ; DOI : 10.4000/ccs.1521 


\title{
L'ENREGISTREMENT DU JARDIN DES PLANTES
}

\author{
Didier ALEXANDRE ${ }^{1}$ \\ Université Paris-Sorbonne
}

La polyphonie s'impose comme le principe narratif dans les romans de Claude Simon, dès Le Tricheur. Louis, Isabelle, Gauthier, Catherine tour à tour racontent, le narrateur, à la manière de Faulkner, variant les voix et les registres. Les Géorgiques reprennent un procédé abandonné depuis La Bataille de Pharsale - pendant la période où les recherches formelles suscitées par l'appartenance déclarée au groupe du Nouveau roman se sont le mieux affirmées et où le vécu (biographique, familial) semble s'être le plus estompé. Avec le retour du fantomatique oncle Charles, dans le roman de 1981, le lien est rétabli avec Histoire et, au-delà, avec La Route des Flandres: le fantôme de Blum erre toujours sur les routes de l'œuvre, voix décalée qui vient provoquer le narrateur. Apparemment, la polyphonie crée un roman dialogique, confrontant sur une question les récits contradictoires, mimant une herméneutique qui échoue.

Jamais pourtant Claude Simon n'avait, comme dans Le Jardin des Plantes, autant confronté les voix. Si le roman de 1997 est un entretien, où se font entendre les voix d'un journaliste et de S., il n'est pas un simple dialogue. À la différence de tous les romans qui ont précédé, les paroles échangées ne sont pas "livrée[s] à l'incohérent, nonchalant, impersonnel et destructeur travail du temps " $(R F$, p. 412): elles sont enregistrées sur un magnétophone. Le récit fait au journaliste, dans l'entretien, devient le récit que nous lisons, comme si lire, c'était écouter la bande sonore. Il en est de cet enregistrement comme de tous les registres de l'œuvre de Claude Simon - cartes postales de la mère d'Histoire et de L'Acacia, registres de l'ancêtre L.S.M. des Géorgiques - et comme des manuscrits chez le 
Pinget de L'Apocryphe: à la relecture, ils sont suspects. Et pourtant, ce ne sont pas les paroles d'un autre qui se font entendre, ce sont les paroles propres, celles de S., qui se font entendre comme différentes, dans leur immédiateté même. Avec Le Jardin des Plantes, le soupçon se déplace des écrits lointains dans le temps et l'espace d'un autre aux paroles immédiatement proches de soi, comme si je était bien un autre, sinon des autres, comme l'écrivait le romancier dans La Corde raide de 1947. La bande sonore de l'entretien révèle que l'écriture de la parole ne comble pas l'intervalle de soi à soi, du même à l'autre, mais au contraire maintient ouverte un entre-deux, un vide où se disent, dans une tension, l'impossibilité de l'achèvement de l'acte littéraire et la nécessité d'une réécriture, ce que Blanchot appelle l'écriture du désastre. Le dispositif du magnétophone, tel le "dispositif Maldoror-Poésies » de Ponge, "permettant [le] sabordage et [le] renflouement à volonté " de la " bibliothèque ${ }^{2}$ ", fait entendre l'échec de l'écriture, l'impossibilité d'être soi dans la voix et l'urgence de substituer au récit d'autres récits - le projet de film à la fin du Jardin des Plantes-, aux fragments d'autres fragments.

Dès que le journaliste sort son magnétophone, S. déclare sa méfiance. Il ne faut pas s'y tromper: la teneur de l'entretien, enregistré, sera aussi une partie du roman - la mise en scène du récit comme l'est, dans La Route des Flandres, l'entretien Blum-Georges.

Vous permettez qu'on enregistre? Sans attendre ma réponse il avait déjà sorti de sa mallette un petit magnétophone qu'il a examiné avant de le poser entre nous sur le coin de la table. J'ai dit Je n'aime pas beaucoup cet instrument. Je n'ai pas le don de parole. C'est même sans doute pourquoi j'écris, je... Il a dit On arrangera tout ça, c'est simplement comme aide-mémoire, après je... Par exemple, excusez-moi, est-ce qu’on ne pourrait pas fermer la fenêtre?... Parce que le bruit de la rue... (JP, p. 957)

Les faiblesses du récit à venir sont clairement affichées: vulnérable au bruit, au parasite qui brouille toute communication, il faut l'isoler de tout contact au réel, du dehors insuffisant, il lui faudra être " arrangé », refait par un autre - le journaliste. S. se définit, avec humour, dans le retrait, non par rapport à un narrateur omniscient, mais par rapport au prophète biblique, qui a le "don de parole » et qui parle la vérité. Chez Simon, le Verbe fait défaut ${ }^{3}$. Cette absence condamne la voix - et l'écriture, nous le verrons - à l'impermanence et à la différence constante. Comme Kyo héros de Malraux, qui, incapable de

2. F. Ponge, Méthodes, dans CEuvres complètes, Gallimard, «La Pléiade », t. 1, 1999, p. 634.

3. C'est pourquoi il faut voir, dans la mention des interrogations de Dostoïevski sur l'existence de Dieu, ou dans l'absence de telles interrogations constatée chez Proust, le reflet des interrogations de Claude Simon lui-même ( $J P$, p. 951). 
reconnaître sa voix lorsqu'il écoute le disque qu’il a enregistré, distingue la voix extérieure qu'il entend de la voix qu'il entend dans sa gorge, S. distingue l'identité de soi à soi ressentie dans l'immédiateté de la parole de la différence de soi à soi ressentie dans la voix enregistrée ${ }^{4}$. Par contre, le journaliste, l'autre, dans l'enregistrement, demeure identique à lui-même.

Il a dit Voyons si ça marche l'autre jour ce machin n’a pas... Il a appuyé sur une touche, puis une autre et j'ai entendu ma voix en sortir - ou plutôt pas exactement: une voix métallique, timbrée, qui n'est pas la mienne ou du moins celle que j'entends quand je parle, disant: ...une sorte d'indignation scandalisée mais sapristi on cherche pour de bon à me tuer et à partir de là... Puis la sienne - mais celle-là c'était la même que celle que j'avais entendue: Excusez-moi vous permettez... (JP, p. 959)

N'est-ce au fond que l'ipséité immédiate qui est inaccessible? Les registres qui conservent les traces écrites du passé - le journal de Rommel, ou les archives des armées conservées au château de Vincennes (JP, p. 1049) - n'enregistrent pas les événements, si enregistrer c'est préserver, intacte, la présence incarnée de soi dans l'écrit. Les mots le peuvent-ils? L'oncle Charles, dans Histoire, ironise sur l'écart qui sépare le vécu sensoriel de l'événement des faits. Le gris symbolise chez Simon le temps où se dissout l'objet pour perdre toute singularité: la trace écrite des archives militaires, dactylographiée " gris sur gris " (JP, p. 1057), subit le même sort, et les mots ne désignent plus aucune réalité.

L'enregistrement vocal échappe-t-il à cette néantisation? Le magnétophone a une spécificité qui le rend supérieur à l'écrit, même immédiat, de l'événement: il saisit la voix, ses rythmes, ses élans et ses défaillances. Il pourrait, mieux que l'écrit susceptible d'être rectifié dans le cours de son élaboration, symboliser cette memoria où la faculté questionnée par Simon, d'un roman à l'autre, rejoint la partie de la rhétorique classique. Car toute bande peut être l'objet d'un retour en arrière et c'est bien ce dispositif de lecture et d'écoute que met en place le roman: il ne s'agit plus dès lors de répéter dans la similitude, et donc de risquer un récit semblable - reprendre la mort du colonel de La Corde raide au Jardin des Plantes en passant par La Route des Flandres, Histoire, L'Acacia -, mais il s'agit de répéter à l'identique. Chaque page, parce qu'elle est censée être entendue du lecteur, est une citation: Claude Simon nous le dit suffisamment, dans l'usage qu'il fait de citations explicites, mises en évidence par la mise en page, qu'elles soient de Dostoïevski (JP, p. 943), de Proust (p. 1000-1001 et 1002), de Flaubert (p. 994),

4. Voir A. Malraux, La Condition humaine dans Euvres complètes, Gallimard, "La Pléiade », 1989, t. 1, p. 520 et p. 548. 
de Rommel (p. 962-963, 987, et passim), de Breton (p. 1086), d'archives du procès de Brodsky (p. 1144), de lui-même:

"Que tout ce qu'il y a d'important en nous se fait à l'aide de tuyaux. » (JP, p. 961) ces choses dont on ne peut parler à une jeune fille [...] sans doute ses différents (quel est ce type qui disait que tout ce qui est important dans la vie se fait à l'aide de tuyaux (Hist., p. 176)

Il y a donc, comme le titrait Libération le 18 septembre 1997, des mémoires de Claude Simon: une mémoire intérieure ( $J P$, p. 960), une mémoire rétinienne, qui a enregistré sur l'œil l'image du " cavalier et [du] cheval semblant basculer " (p. 1070), et une mémoire des textes.

Tout serait simple si Claude Simon s'en tenait à la reprise littérale de textes antérieurs. En fait, Le Jardin des Plantes est tracé d'allées et de paysages déjà parcourus par le lecteur. La lecture est ici lecture d'une mémoire par une mémoire. La scène de la bouche de métro (JP, p. 971) rappelle celle de La Bataille de Pharsale; le "Petit perthuis ", métaphore du sexe féminin "pour Luther" renvoie au texte de Luther placé en exergue de "Chronologie des événements » du même roman. La statue des "Temps futurs" de Perpignan (JP, p. 1168) apparaît dans Histoire. Le lever de soleil aperçu de l'avion en vol est déjà décrit dans Les Corps conducteurs. Gulliver est le titre d'un roman, annoncé dès Le Tricheur (JP, p. 997). La fille du métayer qui séduit « quelque riche propriétaire " $(J P$, p. 1117-1118) est présente dans Le Vent... La liste n'est pas close: un seul groupe nominal - « Riante campagne " placé à la clausule d'un chapitre (JP, p. 1012) - ouvre sur toute l'œuvre passée, de La Route des Flandres aux Géorgiques et à L'Acacia.

Claude Simon ne se pastiche pourtant pas lui-même. Dans ce jeu du même et de l'autre, il veut marquer des différences. La citation n'est pas que simple retour du même: la citation de la mémoire textuelle est une épanorthose, qui permet non pas une fixation, mais un dépassement et un départ vers un texte autre. Le magnétophone symbolise ainsi le mouvement même de l'œuvre simonienne, tournée vers l'arrière pour mieux progresser vers l'avant. On montrerait aisément que la répétition introduit toujours des éléments autres. Par exemple, le récit de l'évasion du stalag diffère de La Route des Flandres (p. 407-408) au Jardin des Plantes (p. 1010) : le lecteur y retrouve les Arabes qui « ramassent des glands " pour les cuire, la sentinelle qui " tourne le dos ", la course "à quatre pattes » dans les " taillis " qui deviennent des « buissons ». Superposée à la scène où Georges fait l'amour à Corinne, la scène d'évasion comporte, dans le roman de 1960, le corps allongé à même le sol, les battements du cœur $(R F$, p. 376377), comme le roman de 1997. L'insensibilité aux déchirures provoquées par 
les fourrés disparaît de la seconde version: ce que Simon met alors en valeur, c'est ce qu'il a ressenti face au risque de mort par "balle " ou à la douleur provoquée par un "coup de baïonnette " (p. 1010). Les circonstances de l'épisode, les sensations physiques, la métaphore animale sont permanentes ${ }^{5}$ : la mise en rapport de l'évasion avec l'amour disparaît pour mieux faire apparaître à travers le souvenir la difficulté de nommer le sentiment ressenti. La superposition des scènes de La Route des Flandres confrontait au risque de mort le furieux désir de vivre: or c'est précisément ce sentiment que $S$. cherche à définir, en réponse au journaliste qui l'interroge sur sa peur ( $J P$, p. 954). L'enquête aboutira à la mélancolie, à cette déclaration:

Jamais je n’avais tant désiré vivre (JP, p. 1122)

La répétition permet ainsi de mieux cerner la différence, qui est double. Elle peut être contextuelle; mais surtout elle dit la reprise de l'interrogation d'un souvenir dont le point central - le désir de vivre, la mélancolie - est toujours différé. La répétition n'a donc de sens que par rapport à un texte passé jugé insuffisant et un mot à trouver. Le Jardin des Plantes insiste sur ce point :

J'ai répété sa question: Comment fait-on pour vivre avec la peur? J’ai dit Hé! On n’a pas le choix!... Il a dit Ce que je voudrais que vous... J'ai dit On a peur, c'est tout. Quoique... Je cherchais mes mots. Je me demandais ce que ceux de "guerre " ou de "peur " pouvaient bien signifier pour lui qui n'était même pas né à cette époque ( $J P$, p. 954)

Le brut n'apparaît plus seulement comme une catégorie esthétique adoptée au lendemain de la Seconde Guerre mondiale en réaction contre l'humanisme discrédité par les horreurs engendrées par le nazisme. Il est l'objet même de l'herméneutique, les "réactions physico-chimiques" (JP, p. 1098): les variations contextuelles - le rejet de toute intrigue romanesque présente dans $L a$ Route des Flandres - disent une progression vers ce point, mais on sait qu'Orion n'atteint jamais la lumière du soleil levant:

c'était encore plus intéressant d'entendre raconter sans ces enjolivements [...] les faits bruts simplement dans leur matérialité parce que Mais $S$. dit que Rien n'est simple, le journaliste disant Bien sûr mais quand même... Je veux dire qu'entre une fiction et le récit, le compte rendu objectif, neutre, d'un événement... (JP, p. 1100)

La relation à un auteur, à travers la citation, joue de même de la différence: non seulement elle est ainsi présentée comme une citation de mémoire, mais elle apparaît autre que ce qu'elle est. La citation faite de Breton (JP, p. 1086), qui

5. Bien évidemment, le lieu de l'évasion a changé: le stalag est situé en France, dans le sud-ouest, et non en Allemagne comme pouvait le laisser penser le texte de 1960. 
est la condamnation de la description, est extraite du Manifeste du Surréalisme ${ }^{6}$. Elle est tronquée: Simon cite de mémoire, ce qui, en soi, confirme notre propos. Breton écrit: " il saisit l'occasion de me glisser ses cartes postales ", et non "L'auteur nous refile ses cartes postales. " De même la citation de Dostoïevski, faite par Breton, differe beaucoup chez Simon. En fait, elle est appropriée par Simon, qui y introduit sa marque:

La petite pièce dans laquelle le jeune homme fut introduit était tapissée de papier jaune: il y avait des géraniums et des rideaux de mousseline aux fenêtres; le soleil couchant jetait sur tout cela une lumière crue... La chambre ne renfermait rien de particulier. Les meubles, en bois jaune, étaient tous très vieux. Un divan avec un grand dossier renversé, une table de forme ovale vis-à-vis du divan, une toilette et une glace adossées au trumeau, des chaises le long des murs, deux ou trois gravures sans valeur qui représentaient des demoiselles allemandes avec des oiseaux dans les mains - voilà à quoi se réduisait l'ameublement.

texte d'André Breton
La petite pièce dans laquelle le jeune homme fut introduit était tapissée de papier jaune; ses fenêtres avaient des rideaux de mousseline; des pots de géranium en garnissaient les embrasures; le soleil couchant l'illuminait à cet instant. Le mobilier, très vieux, en bois clair, était composé d'un divan à l'immense dossier recourbé, d'une table ovale placée devant le divan, d'une table à toilette garnie d'une glace, de chaises adossées au mur et de deux ou trois gravures sans valeur qui représentaient des demoiselles allemandes tenant chacune un oiseau dans les mains, c'était tout.

texte de Claude Simon ${ }^{7}$

En reprenant Breton - au sens de répéter et de corriger -, Simon fait l'éloge de Dostoïevski et le désigne comme un de ses maîtres pour la description. La citation s'inscrit ainsi dans un espace de dialogue, dont la fonction est de définition de soi à travers l'autre. Réécrire Dostoïevski, c'est accomplir un pastiche proustien pour se découvrir soi-même. N'est-ce pas ce qui se produit dans la description d'une scène "vingt ans après l'épisode de la mort du colonel abattu par un sniper" $(J P$, p. 1019) ? Une jeune fille vêtue de rose traverse, à Berlin, « une étendue de ruines » dans le silence, « insolite ", au coucher du soleil. Cette scène, mise en rapport avec le coucher de soleil proustien longuement cité dans le chapitre II, semble la réécriture de Tournesol, poème de Clair de terre de Breton, dont Breton dit, dans « La Nuit du tournesol », publié dans Minotaure ( $\mathrm{n}^{\circ}$ 7, 10 juin

6. Euvres complètes, Gallimard, "La Pléiade ", t. 1, 1988, p. 314.

7. Michel Butor cite ce texte de Crime et châtiment dans « Le roman et la poésie " (Essais sur le roman, Gallimard, "Idées ", 1964, p. 40). Il note que la traduction de Breton est " ancienne ", " peu fidèle ». Il compare cette description à un "passage poétique », un " poème en prose ». Autant dire que roman et poésie se rejoignent. Claude Simon est du côté de la poésie, lui aussi. 
1935), la revue surréaliste dont Claude Simon lisait chaque numéro, qu'il préfigurait la rencontre de sa seconde épouse, Jacqueline Lamba ${ }^{8}$. Cette jeune fille qui sort du métro inverse la prophétie établie par Breton: tandis que le rose est associé au lieu de mort - les cavaliers, à l'aube, sont peints "Regardant venir leur mort en rose" ( $J P$, p. 1068) -, ce que le décor répète dans les ruines, la jeune fille incarne l'objet du désir, la vie. Breton condamne les descriptions qui " cristallisent " " des moments nuls de la vie ${ }^{9}$ ": dans une réécriture à la Breton, Claude Simon cristallise, sur une scène en apparence totalement décontextualisée, le moment fort de sa vie, la révélation du désir de vivre dans l'expérience de la mort. La mélancolie n'est pas nommée: elle reste en-deçà ou au-delà de ce poème en prose, différée. Si la jeune fille disparaît avec le soleil, en épousant son mouvement, Orion, métaphore du romancier, n'atteint pas, nous l'avons rappelé, le soleil levant.

Peut-on mieux approcher cette différence inscrite dans l'écriture et dans la voix? Le récit de la venue du journaliste construit un espace auditif, qui permet à $S$. de se faire écouter et de s'écouter. Cet espace est à la fois ce qui sépare le journaliste de $S$. et ce qui le rapproche de lui, au point que les deux voix se substituent l'une à l'autre: alors qu'au chapitre I, le journaliste est présenté comme "celui à qui [S.] parl[e] dans une langue inconnue" (JP, p. 960), c'est le journaliste qui clôt le chapitre II où sont mis en place simultanément les parcours de Churchill, de Rommel et de S. (p. 1056-1058). Cette proximité précède une collaboration où, par ses questions pressantes, le journaliste arrache à $S$. le terme de mélancolie, et donc le contraint à définir le sentiment qui l'habitait. Il est donc celui qui accouche $S$. de sa vérité. La distance maintenue par Simon, qui note que le journaliste " peut-être " « se fatigue d'entendre S. monologuer " (p. 1097), ou semble "sceptique, réprobateur, irrité " par le terme de mélancolie (p. 1120), interdit toute identification. D'un chapitre à l'autre, les deux interlocuteurs progressent dans la découverte d'un objet inattendu. L'exclamation du journaliste trahit, dans la voix, sa surprise.

Car c'est bien dans la voix que passe la différence: le magnétophone, à l'image de la montre que porte le journaliste « où le temps tourne sur lui-même à différentes vitesses " (p. 1124), symbolise le temps répétitif. Au contraire, la voix humaine est ce qui vient rompre cette répétition, inscrire une différence, qui à son tour deviendra méconnaissable.

8. A. Breton, Euvres complètes, éd. cit., t. 1, p. 187 et p. 1211.

9. Ibid. (Manifeste du surréalisme), p. 315. 
De nouveau je me demandai ce que tout ce que je lui racontais là pouvait bien représenter pour lui. Puisque aucune montre ne peut revenir en arrière. Il y avait seulement le magnétophone qui pouvait. Quoique ce ne fût pas exactement ma voix... (JP, p. 960)

Le Jardin des Plantes souligne ainsi l'importance du récit contre la fiction, et de la diction contre la fiction. Dans la diction, ce qui est recherché, c'est la singularité d'une voix s'emparant d'un texte, c'est donc une chair pénétrant les mots. Claude Simon ambitionne, par son écriture, de retrouver et de faire entendre par les mots cette saveur d'un instant, faite de sensations et d'émotions. C'est dire que toute parole est unique. On comprend que ce soit moins le texte répété que le texte dit qui l'intéresse. Le Jardin des Plantes, en mettant en scène la répétition et la voix, interroge cette diction. Les exemples sont nombreux. Trois suffiront, celui du marché observé par S. depuis la fenêtre de l'appartement qu'il occupe, la statue de Memnon, et enfin la récitation de vers de Racine. Dans ces trois textes, le romancier met en scène la répétition - des jours de marché, de la citation, de hiéroglyphes.

Après le départ du journaliste, S. observe les employés occupés à « démonter les supports des tentes qui abritent les étalages du marché " qui "se tient trois fois par semaine: le dimanche, le mercredi et le vendredi "(p. 1125). Le décompte exact est aux piquets (p. 1126) ce qu'il est à la durée d'une bande de magnétophone. Le chiffre nécessite un travail organisé, une gestuelle minutée: les employés "ne parlent pas, ne s'interpellent pas entre eux, sauf pour crier au conducteur du camion d'avancer au fur et à mesure de leur travail " (p. 1125). Cette organisation du travail a fait aussi disparaître les " cothurnes " dont étaient chaussés jadis les hommes (ibid.). L'installation ou le démontage du marché se répète donc sans théâtralité: ce qui attire pourtant l'attention de S., c'est « au-dessus du bruit de la circulation, [...] le fracas métallique d'un des poteaux (ou d'une des voliges) échappé par mégarde et qui rebondit sur le ciment» (p. 1126). De tels bruits ont, selon S., provoqué les réclamations des riverains. Ainsi la répétition n'est qu'un rituel aseptisé, qui a pour fond une rumeur - le bruit de la circulation - et que vient troubler un bruit. Cette mise en abyme très proustienne de l'écriture désigne la différence comme l'accident qui vient rompre la répétition - le bruit, précisément, dont Michel Serres dit bien dans La Communication qu'il oblige à une réorganisation de l'ensemble de la structure de communication. La description de New York, qu'il faudrait comparer à celles qui sont faites des villes américaines dans Les Corps conducteurs et à la description qu'en fait Sartre, reprend la mécanisation, le fond sonore et audible, et enfin les bruits soudains qui viennent rompre le répétitif: 
les millions de bruits confondus dans une unique et formidable rumeur, vaguement inquiétante, déchirée de loin en loin par le sporadique hululement de sirènes (police, ambulances, pompiers?), croissant, s'exaspérant, strident, décroissant, mourant. Comme des cris de folles, d'oiseaux exotiques, ou les cornes de navires en perdition. Comme si la ville elle-même criait. $(J P$, p. 1066)

C'est dans un tel contexte que peut être située l'écriture. Le souvenir de la mort de la mère s'achève par l'opposition du dedans de la maison - où se trouvent les livres, les œuvres de Rousseau et l'Histoire des Girondins - et du dehors, le jardin, où se trouve l'acacia aux «feuilles semblables à des plumes que les souffles d'air soulèvent et laissent mollement retomber» (p. 1073). La plume prend sens contre une écriture déjà faite, et en réaction à un souffle, celui qui anime le monde, identifié, à l'incipit d'Histoire, à une rumeur de voix.

Le second exemple n'est pas emprunté à l'imaginaire urbain, mais à un imaginaire archéologique. La répétition est présente doublement. Elle est textuelle. En effet, " les deux colosses de Memnon " (JP, p. 1167) font entendre un chant baudelairien, expression du spleen ou de la mélancolie ${ }^{10}$. Leur description reprend des fragments de textes des dernières pages de La Bataille de Pharsale où le romancier compare le souvenir de certains événements vécus pendant la Seconde Guerre mondiale à une fresque sculptée en ronde-bosse érodée par le temps.

Le soleil, la pluie, le gel, la nuit, les aubes, les jours passent tour à tour sur eux sans que leur course se ralentisse. De temps à autre une plaque, un morceau de peau, une joue, une saillie, une épaule, un coude, plus rarement un membre tout entier, s'effrite, tombe en poussière. (BP, p. 735)

Le temps, le vent, le sable, les siècles, l'alternance des brutales chaleurs et des froids brutaux de la nuit les ont amputés d'un bras, d'un pied, parfois d'un membre entier, ont fissuré les blocs de leurs sièges où, dit-on, le vent du désert faisait autrefois, à certaines heures, comme un bruit de harpe. (JP, p. 1167)

Claude Simon met en rapport ces statues avec le temple de "Médinet-Abou ", aux murs extérieurs sculptés de bas-reliefs plus ou moins lisibles et aux colonnades couvertes de hiéroglyphes, qui désignent la répétition.

À l'intérieur alternent de monumentales colonnes rondes ou carrées où la lumière vient s'écraser ou s'amollir, s'accrochant à la profusion des hiéroglyphes profondément gravés qui semblent peupler ces entassements de myriades d'oiseaux, d'un silencieux et assourdissant gazouillis renvoyé par les blocs cyclopéens sous le ciel étouffant et gris du désert. (ibid.)

10. Voir C. Baudelaire, Les Fleurs du Mal, LXXVI ("Spleen») dans Euvres complètes, Gallimard, "La Pléiade ", 1975, t. 1, p. 73. Dans le poème de Baudelaire, ce n'est pas la statue de Memnon qui chante, c'est " un vieux sphinx ». 
Le chant des statues, provoqué dans le corps du souvenir miné par le temps s'élève sur le fond de signes déjà tracés. La différence s'établit entre la lettre écrite et le souffle, symbolisé par le vent du désert.

Dans le dernier exemple, la répétition est assumée dans les références textuelles - Racine, Andromaque, Athalie, Bajazet, Esther - et par les acteurs qui interviennent pour réciter les textes: Antoine $\mathrm{V}$ (itez), dont on sait qu'il a enregistré La Route des Flandres ${ }^{11}$, et Roger C(aillois). L'objet même du débat qui oppose les deux interprètes est l'alexandrin et son allure répétitive. À Roger C. qui reproche au vers sa "monotonie", Antoine V., " en professionnel du théâtre ", oppose une conception éthique et politique du vers:

V. essayant de lui expliquer qu'il en était là du langage comme de ces jardins, ces parcs aux allées taillées en forme de murailles, d'entablements, langue et nature également soumises à la même royale volonté d'ordre, de mesure et de mathématiques (JP, p. 975)

À travers ces jardins, Claude Simon fait référence à son propre jardin - des plantes. Il lui importe moins de résoudre un conflit d'esthétique classique que de montrer que dans ce combat de diction qui oppose V. et C. le plus important est la qualité différentielle d'une voix se manifestant à travers une répétition sur un fond sonore - une rumeur. Or, pendant les récitations, la pluie tropicale se met brusquement à tomber :

se répondaient les voix de héros vêtus de toges ou couronnés de plumes, de reines, de sultanes à brocards et d'empereurs se déchirant dans des palais ou des sérails de carton, faisant se succéder tour à tour en d'élégantes cadences d'élégants sanglots et d'élégantes fureurs sur un fond sonore et puissant de catastrophe. (JP, p. 976)

Le fond sonore se révèle ambigu : il faut distinguer la rumeur-silence qui résulte de bruits culturels qui se neutralisent (bruits de la ville, textes écrits) de la rumeur d'origine matérielle (le souffle du vent). Ainsi, la représentation théâtrale d'une pièce de Picasso mise en scène par Jean-Louis Barrault reprend le schéma que nous avons examiné (texte répété, voix des personnages, " brouhaha de volière, bruyant, pâmé » du public, p. 1154); elle est mise à distance par S. dont la voix se fait entendre à la fin de la séquence: «Il pense: Pauvres bougres, pauvres bougres, pauvres bougres, pauvres bougres..." (ibid.). Car la rumeur que la voix du romancier veut atteindre n'est pas une "voix professionnelle ", celle de V. ou de J.-L. B. Constamment, face au journaliste et son magnétophone, Claude Simon se tourne vers la fenêtre, le dehors, l'air: il observe la place et les oiseaux. Simon aspire, contre les esthétiques constituées, à renouer avec un 
souffle naturel. Au contraire, le journaliste se complaît dans le professionnalisme: S. avec humour constate, après son départ, qu'il a oublié son parapluie, qui devait le protéger contre les averses - tropicales ou autres (p. 1126).

Cette naturalité correspond à une recherche de l'originaire. La mise en scène du Jardin des Plantes, la constitution d'un espace auditif sont proches du dispositif adopté par Samuel Beckett dans La Dernière bande, où le magnétophone et les bandes constituent la mémoire qui peut être déroulée, écoutée et jugée. Les similitudes sont troublantes: chaque écoute est pour Krapp à la fois l'occasion d'un rejet de ce qu'il a été lorsqu'il parlait dans le passé et donc d'une progression - répéter, c'est prendre un départ qui sera source de répétition etc. ${ }^{12}$. L'écoute permet de revenir à des scènes fondatrices: elle est guidée par une quête de l'originaire qui échoue. Les similitudes d'un texte à l'autre sont surprenantes: promenade amoureuse en barque (La Dernière bande, p. 24-26 et p. 32, et $J P$, p. 1120), souvenir de la mort de la mère indissociable de son lieu, la maison (La Dernière bande, p. 19 et JP, p. 1072). Ces scènes originaires sont chez Simon comme chez Beckett des scènes dont l'objet est l'expérience initiatique du désir amoureux - qui échoue, puis réussit $(J P$, p. 1120) - et de la mort (face à laquelle $S$. enfant ne sait comment se conduire, JP, p. 1072-1073). Dans le roman de Simon, ces expériences ne suscitent pas une « viduité » comme chez Beckett (La Dernière bande, p. 19) : l'expérience de la douleur, comme celle du plaisir de l'amour, est associée à la lettre $A$, première lettre s'il en est, où s'inscrit l'origine dans la différence. Dans la répétition du gramme, l'écriture réintroduit constamment la différence, différant toujours le retour à une improbable origine. Novelli illustre parfaitement ce point dans Le Jardin des Plantes, lui qui a rompu avec la rumeur culturelle, est retourné à la rumeur naturelle de la forêt tropicale et s'est constitué son propre alphabet où la lettre réfère à une langue nouvelle, inconnue, tout en portant constamment en elle la différence.

Il a dit Surtout ne bougez pas je risque de vous crever le poumon Je serrais les dents réussis d'abord ce grondement comme un chien puis j'ai cédé tout à coup je me suis entendu crier

$$
\begin{aligned}
& \text { AAAAAAAAAAAA } \\
& \text { AAAAAAAAAAAA } \\
& \text { AAAAAAAAAAAA } \\
& \text { AAAAAAAAAAAA }
\end{aligned}
$$

(JP, p. 962)

12. La Dernière bande suivi de Cendres, Minuit, 1959. Voici comment la répétition suscite la différence: "Sinistres ces exhumations, mais je les trouve souvent [...] utiles avant de me lancer dans un nouveau... (il hésite)... retour en arrière " (p. 16-17). "Viens d'écouter ce pauvre petit crétin pour qui je me prenais il y a trente ans, difficile de croire que j'aie jamais été con à ce point-là. Ça au moins, c'est fini, Dieu merci» (p. 27). 
Une fois même il remplira toute la surface de la toile de lignes de A irrégulièrement tracés, chacun des caractères légèrement différent de celui qui le précède ou le suit non seulement par le dessin (filiforme, gras, soudain plus épais ou plus grand, puis de nouveau mince, boiteux) mais encore par la couleur [...] il gravera les lignes superposées de lettres irrégulières, ondulant comme un cri, se répétant, jamais identiques:

AAAAAAAAAAAAAAAAAAAAAAAAAAAAAAA

AAAAAAAAAAAAAAAAAAAAAAAAAAAAAAA

AAAAAAAAAAAAAAAAAAAAAAAAAAAAAAA

(JP, p. 1080 ; voir cahier iconographique)

Dans La Route des Flandres, le cri et les hurlements de l'orgasme font entendre le même son, «sa bouche faisant Aaah aaaaaaaah» (p. 396).

L'érotisme simonien rejoint ainsi l'expérience de la douleur et de la mort: c'est dans le corps propre ou le corps de l'autre auquel le sujet veut se fondre que la coïncidence de soi à soi peut être donnée. À partir du moment où il y a graphème, ou enregistrement, le sujet peut se voir ou s'entendre objectivé au dehors de lui-même sous forme de signes réduits au statut d'indices. Tout texte chez Simon a valeur d'indice par rapport à un vouloir-dire présent. Ce que recherche Simon, c'est donc bien le phonème, c'est-à-dire le son de la phoné antérieur à toute signification, ce moment où la voix s'entend parlant. L'oralité de l'œuvre simonienne, son retour constant sur ce qui a été écrit, son interrogation des textes conçus comme des traces se comprennent en fonction de cette quête de "l'âme du langage ", de " l'acte vivant ", " de l'intimité de la vie avec elle-même ${ }^{13}$ ». C'est cette présence de soi à soi dans le fait de s'entendre parler qui est la condition de possibilité d'une expression qui dise pleinement son objet: à travers la chair, l'être du sujet et l'être du monde coïncident, dans l'instant. C'est pourquoi Le Jardin des Plantes peut être un monologue (JP, p. 1097) et un récit qui, une fois achevé, objectivé dans la bande, devient le lieu d'une différence.

Cette lecture phénoménologique rejoint la lecture blanchotienne qui peut être donnée du Jardin des Plantes. L'œuvre écrite, conçue comme répétition et différence, n'est à chaque fois qu'un livre. Tandis que la voix se situe dans l'espace littéraire, à la recherche de ce point, désigné par la mémoire corporelle chez Simon, d'où viennent tous les livres, que nous désignons par l'origine, le texte est un produit achevé, une figure de l'œuvre idéale à accomplir. Une semblable lecture rend compte du mouvement global du roman. La mise en page tout à fait nouvelle des trente-trois premières pages du roman mime la juxtaposition mémorielle des souvenirs: la page est bien cet espace antérieur aux mots et aux textes qui viennent, dans l'espacement, se mettre en rapport. Progressivement,

13. J'emprunte ces expressions à J. Derrida, La Voix et le phénomène, PUF, 1967, chap. VI, « La voix qui garde le silence ", p. 87. 
l'œuvre se déploie et se construit à partir de ce "lieu d'extrême vacance ", où les mots apparaissent et disparaissent ${ }^{14}$. Le roman, dans sa fragmentation, loin d'être l'acceptation d'un désordre, est une mise en ordre où les blancs isolent les fragments. Les parcours indiqués à l'intérieur du roman ne suppriment pas les écartements: mais ils laissent supposer l'existence, sous-jacente, d'un " espace corrélatif, supplémentaire, secondaire " qui est aussi, paradoxalement, le blanc où tout se dissout ${ }^{15}$. Le passage du magnétophone - le successif, grevé par la différence - au cinéma, dans Le Jardin des Plantes, marque une tentative de mieux dominer l'espace littéraire: la projection simultanée sur des écrans différents, pratiquée par Abel Gance dans son Napoléon ou par Claudel dans ses projets de mise en scène du Livre de Christophe Colomb, apparaît comme une solution susceptible de réduire l'écart qui existe d'un fragment à l'autre $(J P$, p. 1055). Le scénario cinématographique qui clôt le roman, sans parvenir à réaliser ce projet, confirme ce déplacement de l'espace auditif à l'espace visuel du livre. La pensée de Blanchot permet encore de comprendre la constante présence, dans les fragments simoniens, du texte répétitif, de la voix, et de leur confrontation à la rumeur. Chez Blanchot, comme chez Simon, le silence est rumeur, totalité de bruits singulièrement identifiables, mais dont l'ensemble forme un tout indistinct, neutre. C'est cette " rumeur initiale » que le texte désire approcher ${ }^{16}$. Le sentiment de mélancolie est indissociable de cette rumeur:

Hésitant de nouveau (et pendant un moment il peut de nouveau percevoir ce même indifférent et menaçant grondement, cette espèce de bruit de fond, cette rumeur étale, sans plus de consistance qu'une faible et unique vibration dans quoi vient se confondre toute l'agitation du dehors, se neutraliser toute la violence, les passions, les désirs, les peines, les terreurs), et à la fin il dit Mélancolie (JP, p. 1119-1120)

L'ultime paradoxe du Jardin des Plantes - mais aussi, comme nous y invitent les répétitions, des romans antérieurs - réside dans le statut générique du roman: il n'est pas roman, au sens fictionnel du terme, il est plus proche du poème, par sa démarche formelle, sa quête phénoménologique, son interrogation sur le langage et ses enjeux ontologiques.

Peut-être est-ce là la raison qui pousse Claude Simon à revenir sur des questions soulevées par la diffusion, lors du colloque de Cerisy-la-Salle organisé par

14. M. Blanchot, Le Livre à venir, Gallimard, 1959, p. 345. Blanchot commente alors Un coup de dés de Mallarmé: "l'espace antérieur que supposent ces rapports et dont le mouvement originaire rend seul possible le langage comme déploiement. " Je rappelle cette définition du livre que donne Mallarmé: "Le livre, expansion totale de la lettre, doit d'elle tirer, directement, une mobilité et spacieux, par correspondances, instituer un jeu » (Euvres complètes, Gallimard, "La Pléiade », 1945, p. 380).

15. M. Blanchot, Le Pas au-delà, Gallimard, 1984 [1973], p. 72.

16. M. Blanchot, L'Espace littéraire, Gallimard, 1982 [1955], p. 79. 
Jean Ricardou et Françoise Van Rossum-Guyon ${ }^{17}$ en juillet 1971, de documents qui attestaient la réalité des faits racontés dans La Route des Flandres ou qui étaient à l'origine de certaines descriptions d'Histoire (billets de banque). Le Jardin des Plantes revient ainsi, par cette autre reprise, ou répétition, d'un débat enregistré au magnétophone, sur la question du Nouveau roman, puisque l'entretien faisait suite à la communication de Jean Ricardou intitulée « Le Nouveau roman existet-il ? " Ce que Jean Ricardou rejette dans la discussion, le fait qu'un officier ait adressé à Claude Simon une lettre où il disait avoir vécu les événements relatés dans La Route des Flandres et donc se soit reconnu dans la fiction à la suite d'un effet de réel, c'est "l'illusion de projection " fondée sur "l'illusion référentielle ${ }^{18} »$. Ce double rejet s'explique par la place prépondérante occupée dans la théorie de Jean Ricardou par la production du texte et la condamnation de l'expression et de la représentation caractéristiques de l'écriture romantique.

Avec l'Expression-Représentation, il y a le malheur d'une discordance entre le sens institué et le langage. Celui-ci, dans la mesure où il est intermédiaire, tend incessamment à déformer le quelque chose à dire. [...] Avec la production, le rapport du sens et du langage se renverse: le sens n'est plus une institution préalable qu'il importe de faire paraître, c'est, désormais problématique, l'effet des agencements d'un langage calculé ${ }^{19}$.

La réponse apportée par Claude Simon à ces points du débat est très nuancée. En effet, si le sentiment de mélancolie renvoie bien à une expression de soi, Claude Simon prend soin de le distinguer du sentiment post-romantique et pré-raphaélite $(J P$, p. 1122). La vitalité qu'il suppose nous renverrait plutôt à une éthique et une esthétique nietzschéennes. La réponse apportée à la question de la représentation est plus complexe. En effet, lorsqu'il évoque, par exemple, le projet de scénario final, Claude Simon rappelle que les cavaliers avaient bien enduit de boue leurs casques et coquilles de sabre pour se dissimuler à l'ennemi et que ce détail doit disparaître dans le film, car il serait jugé «l'œuvre maladroite d'un accessoiriste afin de faire plus "vrai" " $(J P$, p. 1178). Outre une réponse ironique apportée à Ricardou - faire vrai, c'est faire trop vrai -, le romancier apporte sa propre contribution à la question de la représentation: il ne s'agit évidemment pas de créer une vérité du texte, qui serait conforme à une attente du spectateur ou du lecteur, et qui donc préexisterait au texte

17. Voir $J P$, p. 1161-1163, reprise des dialogues enregistrés au magnétophone à Cerisy dans une discussion à laquelle Claude Simon, qui n'était pas encore arrivé, ne participa pas (Nouveau Roman, hier, aujourd'hui, UGE, «10/18 », 1972, t. 1, p. 28-31).

18. Nouveau Roman, hier, aujourd'hui, éd. cit., t. 1, p. 28. La lettre du colonel est citée par Simon (JP, p. 1161) et reproduite dans Entretiens, n 31 ("Claude Simon »), Montpellier, Éditions Subervie, 1972, entre p. 152 et p. 153.

19. Nouveau Roman, hier, aujourd'hui, éd. cit., t. 1, p. 23. 
à la fois chez l'écrivain et son public, mais de créer par l'écriture un texte qui re-présente, restitue la présence simultanée dans l'écrivain d'un ensemble de souvenirs, de sensations, d'émotions. Le roman simonien est donc expressif de celui qui l'écrit, et recourt à des documents éventuels qui, au même titre que les souvenirs, sont des matériaux. S'il y a illusion, elle n'est pas référentielle: par contre, rien n'interdit au lecteur de partager l'émotion qui habite le texte.

Cette réponse apportée à Jean Ricardou vise aussi à protéger l'indépendance de l'écrivain. Le Nouveau roman fut présenté comme un groupe dont Jean Ricardou définissait l'orthodoxie. Certains intervenants du colloque de Cerisy de 1971 soulignèrent ce point - par exemple Nathalie Sarraute ${ }^{20}$. Claude Simon reprend, sur le mode ironique, le questionnement de Ricardou:

S. n'avait-il pas enfreint les principes de base d'un certain mouvement littéraire? En rendant public un tel document, $S$. ne contrevenait-il pas aux théories dont se réclamaient les adeptes de ce mouvement? (JP, p. 1161)

En traçant dans Le Jardin des Plantes le parcours très libre, politiquement, de l'écrivain, Claude Simon affiche son indépendance à l'égard du Nouveau roman. Une simple relecture du texte suffit pour comprendre que l'être même de Simon se confond avec la liberté et qu'il rejette toute forme de théorie préalable au texte - ce qui serait pour lui le Nouveau roman. S. en URSS refuse de signer un document de complaisance à l'égard du pouvoir politique (JP, p. 974), S. aide les membres d'un réseau clandestin - les Nouveaux Romanciers? - sans participer activement à leur action, montrant peu d'intérêt pour des codes secrets qui lui sont expliqués (JP, p. 1147-1148), S. reproduit des documents du ministère des armées classés «REPRODUCTION INTERDITE » (représentation interdite). Peut-être faut-il aussi replacer le procès de Brodsky dans le contexte de cette interrogation sur les contraintes politiques exercées sur l'écrivain (JP, p. 1144). Dans un entretien avec Mireille Calle-Gruber de 1982, Alain Robbe-Grillet rappelle que « ce qu'[il a] réclamé pour le romancier, c'est la liberté » et va à l'encontre des " normes fixées ${ }^{21}$ ". La volonté de Claude Simon ne differe pas, farouche: en cela, Le Jardin des Plantes demeure un Nouveau roman, car il plaide pour l'homme et pour la liberté, contre toute règle esthétique, éthique, politique pré-existant au texte. Ainsi se justifie encore, au regard de l'histoire littéraire, un roman qui se construit dans la différence et la recherche constante de ce qu'il est par la traversée de ce qu'il a été.

20. Voir Nouveau Roman, hier, aujourd'hui, éd. cit., t. 2, "Ce que je cherche à faire ». 21. Micromégas, n² 20, spécial Nouveau Roman, Rome, 1981, p. 7. 